Trauma Berufskrankh 2008 · 10 [Suppl 2]:252-255

Online publiziert: 20. April 2008

(c) Springer Medizin Verlag 2008
DOI 10.1007/s10039-007-1330-4

\author{
A. Lohsträter ${ }^{1}$. J. Moock ${ }^{2} \cdot$ S. Germann ${ }^{1} \cdot$ T. Kohlmann ${ }^{2} \cdot$ A. Ekkernkamp ${ }^{3,4}$ \\ ${ }^{1}$ VBG (Verwaltungs-Berufsgenossenschaft), Erfurt \\ ${ }^{2}$ Institut für Community Medicine, Universitätsklinikum, Greifswald \\ ${ }^{3}$ Unfallkrankenhaus, Berlin \\ ${ }^{4}$ Abteilung für Unfall- und Wiederherstellungschirurgie, \\ Universitätsklinikum, Greifswald
}

\section{Bedeutung der postoperativen Übungsbehandlung für das Outcome der distalen Radiusfraktur}

- kaufmännische oder gewerbliche Arbeitnehmer,

- Zeitarbeitnehmer,

- Umschüler und

- sonstige Versicherte der VBG

und deren Outcome untersucht; Art Umfang und Ausmaß des Einsatzes von Übungsbehandlungen standen dabei eher im Hintergrund (z. B. $[3,4,5,7]$ ). Therapeutische Lücken zwischen der Akut- und der Nachbehandlung, die Auswirkungen auf das Outcome haben können, sind in der Praxis nicht unbekannt.

Die aktuelle Übersicht des Cochrane Reviews zur Rehabilitation bei Erwachsenen mit Frakturen des distalen Radius kommt zu dem Ergebnis, dass die geeignete Form der Nachbehandlung nicht mit ausreichender Evidenz erwiesen ist [1].

In der vorgestellten Arbeit wurde u. a. die Frage untersucht, welchen Stellenwert die strukturierte, postoperative Übungsbehandlung auf das Outcome der distalen Radiusfraktur, definiert als die Höhe der Minderung der Erwerbsfähigkeit (MdE), hat.

\section{Material und Methode}

\section{Patienten}

Die Studie wurde in der Zeit von 10/2002o1/2006 als randomisierte Studie mit 198 Patienten durchgeführt. Als Einschlusskriterien für die Probanden waren festgelegt: aus Sachsen-Anhalt und Thüringen im Alter zwischen 16 und 65 Lebensjahren mit einer isolierten distalen Radiusfraktur.

Kontrollgruppe. Die Steuerung und Überwachung des Heilverfahrens sowie das Verordnen von Art, Intensität und Ausmaß der Übungsbehandlung lagen ausschließlich in der Hand des behandelnden D-Arztes.
Interventionsgruppe. Nach Eingang des Durchgangsarzt- und ggf. des Operationsberichts wurden die Unterlagen dem beratenden Handchirurgen übermittelt. Dieser gibt innerhalb von $24 \mathrm{~h}$ eine dezidierte Behandlungsempfehlung für den weiterbehandelnden Arzt ab. Auf dem standardisierten Bogen können einzelne Behandlungsmaßnahmen (BGSW, EAP, KG, Ergotherapie usw.) hinsichtlich Beginn, Art und Dauer vorgeschlagen werden. Voraussichtliche Arbeitsunfähigkeitszeiten werden prognostisch angegeben [2]. Die ausführenden Reha-Manager leiten diese Empfehlungen an den Behandler weiter, informieren und beraten die Patienten entsprechend und über-

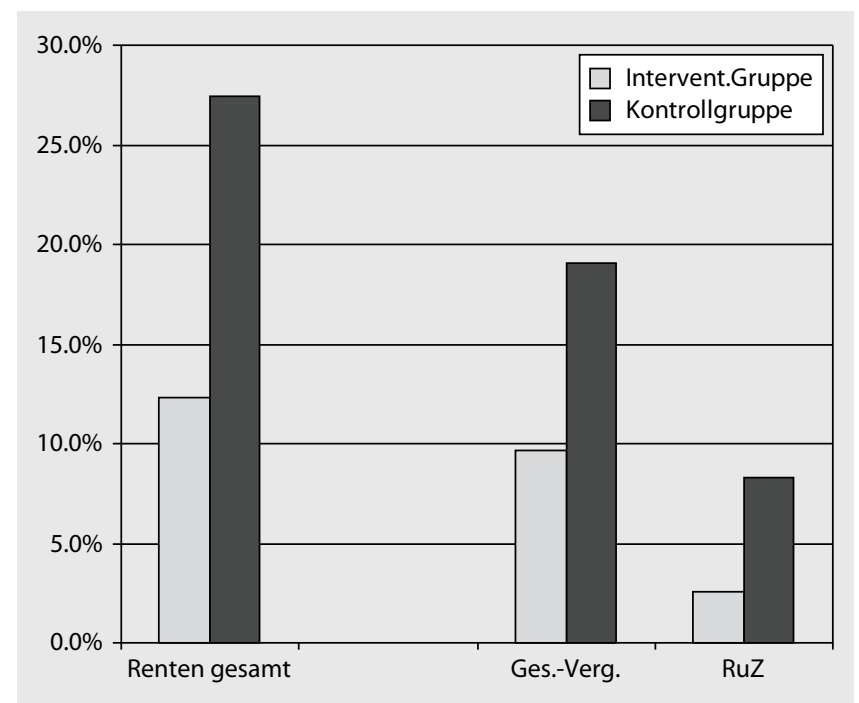

Abb. $1 \varangle$ Übersicht der zu gewährenden Renten, Ges.-Verg. Gesamtvergütung, RuZ Rente auf unbestimmte Zeit, Intervent.Gruppe Interventionsgruppe 
wachen die Umsetzung. Legen die behandelnden Ärzte alternative Behandlungsstrategien vor, stimmen die Reha-Manager diese mit dem beratenden Handchirurgen $\mathrm{ab}$ und koordinieren das abschließende Vorgehen.

\section{Auswertung}

Zur Randomisierung wurden ein Set von Zufallszahlen computergestützt extern generiert und die Randomisierungstabelle dort vorgehalten. Zur Beantwortung der Studienfrage und der aufgestellten Hypothesen wurden die Frakturen entsprechend der Klassifikation der AO klassifiziert sowie die Daten der durchgeführten Übungsbehandlungen erhoben. Diese Werte wurden mit dem Outcome, definiert als Höhe und Häufigkeit einer Minderung der Erwerbsfähigkeit (MdE), der jeweiligen Studienarme verglichen.

Die Signifikanztests wurden mit einem Signifikanzniveau von $5 \%$ durchgeführt.

\section{Ergebnisse}

\section{Studiengröße}

Im Zeitraum von Oktober 2002 bis einschließlich Januar 2006 konnten $380 \mathrm{~Pa}$ tienten randomisiert werden. Aufgrund unterschiedlicher Drop-Outs bildeten 198 Patienten, aufgeteilt in beide Gruppen, die empirische Basis der Studie.

\section{Demografie}

Die auf die Studienarme bezogenen Verteilungen von Alter, Geschlecht und Frakturklassifikation waren homogen und wiesen keine signifikanten Unterschiede zwischen den Gruppen auf.

\section{Übungsbehandlung im Gruppenvergleich}

Die Studienergebnisse zeigten die Verordnung von Maßnahmen der Physiotherapie über die Gruppen hinweg als bekannte Größe. Das Verordnungsverhalten von Maßnahmen der Ergotherapie war gruppenspezifisch unterschiedlich. Auffällig war, dass auch in der Interventionsgruppe Fallgestaltungen vorkamen, in denen

Trauma Berufskrankh 2008 · 10[Suppl 2]:252-255 DOI 10.1007/s10039-007-1330-4

(c) Springer Medizin Verlag 2008

\section{A. Lohsträter · J. Moock · S. Germann · T. Kohlmann · A. Ekkernkamp Bedeutung der postoperativen Übungsbehandlung für das Outcome der distalen Radiusfraktur}

\section{Zusammenfassung}

In einer prospektiv randomisierten Studie wurde die Wertigkeit einer konsequenten und strukturierten Übungsbehandlung auf das Outcome der distalen Radiusfraktur untersucht. Von 380 konsekutiven Patienten konnten 198 in die Studie eingeschlossen und einer Interventions- (IG, gezieltes Management) oder Kontrollgruppe (KG, D-ArztVerfahren) randomisiert zugeteilt werden. In der IG erhielten die Behandler Empfehlungen zu Art, Umfang und Ausmaß der durchzuführenden Übungsbehandlung. Die Nachbehandlungsempfehlung wurde durch die VBG in ihrer Umsetzung begleitet. In der IG ergab sich ein geringeres $\mathrm{Ma} ß$ an verordneter Therapie. Diese wurde jedoch strukturierter und in kürzerer Zeit erbracht. Bezüglich des Outcomes, gemessen in der Minderung der Er- werbsfähigkeit (MdE), ergab sich, bei kürzerer Arbeitsunfähigkeitszeit, in der IG nur in 12,3\% der Fälle eine MdE, in der KG, trotz scheinbar längerer Nachbehandlungsphase, dagegen in $27,4 \%$. Das Outcome war somit in der IG signifikant besser, wobei keine höheren Therapiekosten zu beobachten waren. Die strukturierte Nachbehandlung im Sinne einer kombinierten Therapie wird in ihrer Bedeutung noch unterschätzt, daher scheint eine unterstützende Steuerung geboten. Sie sichert im Zusammenwirken aller Beteiligten einen gröBeren Behandlungserfolg.

\section{Schlüsselwörter}

Distale Radiusfraktur · Outcome . Strukturierte Nachbehandlung . Ergotherapie $\cdot$ Rehabilitation

\section{Importance of structured post-operative treatment on the outcome after distal fracture of the radius}

\section{Abstract}

The value of consequent and structured tutorial treatment on the outcome of distal radius fractures was examined within a prospective randomized study. Due to assessment criteria, 198 out of 380 consecutive distal fracture patients could be included in the study and randomly assigned to either an intervention group (focused management) or a control group (transition physician process). Recommendations about character, extent and degree of the exercise treatment conducted were given to therapists, in the intervention group post-treatment recommendations were carried out according to standard practice. In the interventions group there was a low degree of prescribed therapy, however, these therapies were carried out in a more structured way and within a shorter time frame. The outcome revealed that only $12.3 \%$ of cases treated within the intervention group, as measured by the reduction of earning capacity, showed a reduction despite apparently shorter periods of work incapacity. In contrast, a reduction of earning capacity was found in $27.4 \%$ of the cases treated within the control group despite apparently longer post-treatment periods. Working incapacity time periods and the outcome (reduction of earning capacity) were significantly shorter and better within the intervention group and higher therapy costs were not observed The relevance of structured post-treatment in terms of a combined therapy has therefore been underestimated and supportive control appears to be necessary. Combining both therapies assures higher treatment success in cooperation with all parties involved.

\section{Keywords}

Distal radius fracture $\cdot$ Outcome $\cdot$ Structured post-treatment - Occupational therapy . Rehabilitation 
Tab. 1 Verordnungsverhalten von Physiotherapie in IG und KG

\begin{tabular}{|llllll} 
Physiotherapie & IG & & KG & & $\begin{array}{l}\text { U-Test nach } \\
\text { Mann-Whitney }\end{array}$ \\
& $\begin{array}{l}\text { Mittel- } \\
\text { wert }\end{array}$ & $\begin{array}{l}\text { Standard- } \\
\text { abweichung }\end{array}$ & $\begin{array}{l}\text { Mittel- } \\
\text { wert }\end{array}$ & $\begin{array}{l}\text { Standard- } \\
\text { abweichung }\end{array}$ & $\begin{array}{l}\text { p-Wert } \\
\text { Beginn [Tage nach Unfall] }\end{array}$ \\
34,4 & 16,2 & 32,2 & 18,8 & 0,303 \\
\hline Ende [Tage nach Unfall] & 78,3 & 48,4 & 124,9 & 169,9 & 0,010 \\
\hline Verordnete Einheiten & 28,8 & 29,1 & 46,0 & 52,9 & 0,068 \\
\hline Kosten [EUR] & 354,43 & 348,58 & 576,33 & 603,59 & 0,006 \\
\hline
\end{tabular}

Tab. 2 Verordnungsverhalten von Ergotherapie in IG und KG

\begin{tabular}{|llllll} 
Ergotherapie & IG & & KG & & $\begin{array}{l}\text { U-Test nach } \\
\text { Mann-Whitney }\end{array}$ \\
& $\begin{array}{l}\text { Mittel- } \\
\text { wert }\end{array}$ & $\begin{array}{l}\text { Standard- } \\
\text { abweichung }\end{array}$ & $\begin{array}{l}\text { Mittel- } \\
\text { wert }\end{array}$ & $\begin{array}{l}\text { Standard- } \\
\text { abweichung }\end{array}$ & p-Wert \\
Beginn [Tage nach Unfall] & 44,2 & 19,3 & 100,1 & 102,7 & 0,016 \\
\hline Ende [Tage nach Unfall] & 85,1 & 49,5 & 139,0 & 112,4 & 0,028 \\
\hline Verordnete Einheiten & 27,7 & 63,4 & 32,5 & 32,0 & 0,022 \\
\hline Kosten [EUR] & 426,73 & 322,21 & 689,86 & 537,44 & 0,031 \\
\hline
\end{tabular}

weder Physio- noch Ergotherapie verordnet worden waren.

In der Kontrollgruppe wurden in 18 von 84 Fällen der vorliegenden Radiusfrakturen weder Physiotherapie noch Ergotherapie verordnet und durchgeführt. Ein Schwerpunkt hinsichtlich der Frakturklassifikationen war nicht festzustellen. Lediglich bei den $\mathrm{C}_{3}$-Frakturen wurde immer Physiotherapie verordnet.

In der Interventionsgruppe wurde in 17 von 114 Fällen keinerlei Übungsbehandlung verordnet. Ein Schwerpunkt der ohne Übungsbehandlung gebliebenen distalen Radiusfrakturen war bei den A2- und B1-Frakturen festzustellen.

Während der Beginn der Physiotherapie, gemessen in Tagen nach dem Unfall, in beiden Gruppen nahezu gleich war, waren beim Ende der Übungsbehandlung deutliche Unterschiede festzustellen. Ebenso deutlich unterschieden sich die Anzahl der verordneten Einheiten in den Gruppen, auch wenn hier das Signifikanzniveau nicht ganz erreicht wurde. Bei den Kosten hingegen waren signifikante Unterschiede zwischen den Gruppen zugunsten der Interventionsgruppe zu erheben. Die Differenz zwischen den Gruppen betrug 221,90 EUR. Festzuhalten bleibt, dass in der Interventionsgruppe deutlich mehr Therapie in kürzerer Zeit stattfand.

In - Tab. 1 ist das Verordnungsverhalten bezüglich Übungsbehandlung, so weit diese verordnet wurde, in der Kontroll- und Interventionsgruppe dargestellt.
Als Therapieeinheit war definiert, dass an diesen Tagen entsprechende Übungsbehandlung stattgefunden hat.

Während sich die Verordnung von Physiotherapie noch als bekannte Größe darstellte, waren bei dem Verordnungsverhalten der Ergotherapie massive Unterschiede zwischen der Kontroll- und der Interventionsgruppe zu beobachten. Der Beginn der, so weit überhaupt verordneten, Ergotherapie in der Kontrollgruppe lag im Mittelwert bei 100 Tagen und damit über 50 Tage später als in der Interventionsgruppe. Ähnlich verhielt es sich mit dem Ende der verordneten Ergotherapie. Auch bei der Anzahl der verordneten Einheiten Ergotherapie sowie bei der Kostenstruktur waren signifikante Vorteile zugunsten der Interventionsgruppe zu beobachten (- Tab. 2).

Eine kombinierte Verordnung von Physiotherapie und Ergotherapie im Sinne einer multimodalen Nachbehandlungsstrategie war in der Kontrollgruppe die Ausnahme. In der Interventionsgruppe war dies wesentlich häufiger der Fall (56\%). Die Bereitschaft zur kombinierten Verordnung von Physio- und Ergotherapie nahm mit der Dauer der Studie zu.

\section{Minderung der Erwerbsfähigkeit}

Das Outcome, gemessen in der Minderung der Erwerbsfähigkeit (MdE), zeigte, trotz kürzerer Arbeitsunfähigkeitszeiten in der Interventionsgruppe, in deutlich weniger Fällen eine MdE von mindestens 20 von Hundert. In der Kontrollgruppe war in $27,4 \%$ eine Rente, wenn $z$. T. auch nur vorübergehend, festzustellen; in der Interventionsgruppe hingegen nur in $12,3 \%$ der Fälle. Davon waren in der Kontrollgruppe $19,1 \%$ und in der Interventionsgruppe 9,7\% Gesamtvergütungen. Berufsgruppenbezogene Schwerpunkte waren dabei nicht festzustellen. Renten auf unbestimmte Zeit waren in der Kontrollgruppe in $8,3 \%$ und in der Interventionsgruppe in 2,6\% der Fälle zu gewähren (• Abb. 1).

\section{Diskussion}

Die Verordnung von Physiotherapie bei der Nachbehandlung der distalen Radiusfraktur stellt sich als bekannte Größe dar, die Verordnung von Ergotherapie dagegen scheint ohne steuernde Eingriffe eher die Ausnahme zu sein.

Die aufgestellten Hypothesen hinsichtlich der Intensität der Übungsbehandlung in der Interventionsgruppe und der daraus resultierenden höheren Kosten konnten nicht bestätigt werden. In der Interventionsgruppe wurde über die gesamte Behandlungszeit durchschnittlich weniger Übungsbehandlung erbracht als in der Kontrollgruppe. Betrachtet man Intensität als Einheiten von Übungsbehandlung (kombinierte Physio- und Ergotherapie) innerhalb eines bestimmten, möglichst kurzen, Zeitraums, bestätigen die gefundenen Ergebnisse eine intensivere Nachbehandlung in der Interventionsgruppe. Über den gesamten Fallverlauf wurde in der Kontrollgruppe mehr an Übungsbehandlungen verordnet als in der Interventionsgruppe, allerdings über einen längeren Zeitraum. Damit waren die Kosten der Übungsbehandlung in der Interventionsgruppe nicht höher. Die zugunsten des Handreha-Managements der VBG in Kauf genommene Hypothese der höheren Kosten für Übungsbehandlungen ließ sich somit nicht bestätigen.

Auffällig war, dass in der Anfangsphase der Studie selbst in der Interventionsgruppe, trotz Empfehlung des Kostenträgers, das Verordnungsverhalten hinsichtlich durchzuführender Ergo- oder kombinierter Therapie zurückhaltend war. 
Das Outcome, definiert als Minderung der Erwerbsfähigkeit (MdE), war in der Interventionsgruppe signifikant besser als in der Kontrollgruppe. Letzteres entsprach weitestgehend auch den bisher publizierten Feststellungen anderer Unfallversicherungsträger [6]. Die strukturierte Nachbehandlung im Sinne einer Kombination aus Physio- und Ergotherapie sowie abschwellender Maßnahmen scheint in ihrer Wirkung noch unterschätzt zu werden. Die Ergebnisse in der Interventionsgruppe belegen allerdings eindrucksvoll, welches Kosten-Nutzen-Verhältnis mit dieser Art der kombinierten Therapie $\mathrm{zu}$ erreichen ist.

Die hypothetisch angenommenen niedrigeren Gesamtfallkosten in der Interventionsgruppe ließen sich bereits ohne ökonomische Erhebungen durch die kürzeren Arbeitsunfähigkeitszeiten und niedrige Rentenquoten verifizieren. Spezifische ökonomische Analysen haben zu folgen und sind zu publizieren.

Die externe Validität im Sinne einer Generalisierbarkeit der Ergebnisse lässt sich aufgrund der Studiengröße durchaus ableiten. Hohe Signifikanzen liegen vor. Das Studiendesign und die Durchführung der Studie erscheinen zur Beantwortung der gestellten Frage belastbar.

Die Ergebnisse zeigen einen hohen Wirkungsgrad des Handreha-Managements der VBG.

\section{Fazit}

Auf die im Rahmen des berufsgenossenschaftlichen Heilverfahrens bestehenden Regelungen kann als Grundvoraussetzung nicht verzichtet werden. Sie erreichen allerdings einen noch höheren Wirkungsgrad, wenn der das Heilverfahren führende UV-Träger Managementverfahren weiterentwickelt und mit seinen entsprechend qualifizierten Reha-Managern aktiv zielgerichtete Handlungsbeiträge dazu erbringt.

Das Outcome, gemessen als Minderung der Erwerbsfähigkeit (MdE), war in der Interventionsgruppe signifikant besser. Höhere Therapiekosten waren, auch ohne Budgetschranken, in der Interventionsgruppe nicht zu beobachten. Untersuchungen, die die Intensität, die Dauer und den Wert von Physio- und Ergothe- rapie beleuchten, sind bisher nicht publiziert. Die strukturierte Nachbehandlung im Sinne einer kombinierten Therapie wird in ihrer Bedeutung daher noch unterschätzt. Insbesondere bei der Verordnung von Ergotherapie fallen deutliche Defizite auf. Eine unterstützende Steuerung scheint daher geboten. Als Therapieempfehlungen hinsichtlich Beginn, Art und Dauer der Nachbehandlung bieten sich dazu z. B. die Standards für Heilverfahren und Rehabilitation [8] der VBG an. Die Kombination beider Therapieverfahren sichert im Zusammenwirken aller Beteiligten einen größeren Behandlungserfolg. Die interdisziplinäre Zusammenarbeit zwischen UV-Trägern, Ärzten und Therapeuten ist dabei in einem höheren Maß gefordert als bisher.

\section{Korrespondenzadresse}

\section{Dr. A. Lohsträter}

VBG (Verwaltungs-Berufsgenossenschaft), Koenbergkstraße 1, 99084 Erfurt

Axel.Lohstraeter@vbg.de

Interessenkonflikt. Der korrespondierende Autor gibt an, dass kein Interessenkonflikt besteht.

\section{Literatur}

1. Handholl HHG, Madhok R, Howe TE (2006) Rehabilitation for distal radial fractures in adults. Cochrane Rev 3: CD003324

2. Lohsträter A, Schmidt J (2002) Rehabilitationsmanagement bei Hand- und Handgelenksverletzungen aus Sicht des UV-Trägers. Trauma Berufskrankh 4: 163-167

3. Oestern H (2003) Aktueller Stand der Behandlung von Radiusfrakturen. Trauma Berufskrankh [Suppl 1] 5: S22-S25

4. Pabst T, Uzdil T, Winker KH (2003) Die palmare Plattenosteosynthese bei der distalen Radiusfraktur. OP J 19: 42-50

5. Pilz F, Lindemann-Sperfeld L, Winter S et al. (2000) Distale Radiusfrakturen - Behandlungskonzepte und Erfahrungen. Trauma Berufskrankh 2: 313319

6. Radek E, Köhler T, Münch K et al. (2003) Verletztenrenten auf unbestimmte Zeit nach distalen Radiusfrakturen. Trauma Berufskrankh 5: 253-259

7. Siebert H, Klonz A (2005) Distale Radiusfraktur. Unfallchirurg 108: 135-154

8. VBG (2007) Standards für Heilverfahren und Rehabilitation. VBG, Hamburg, S 59-64 